\title{
The CYP2D6B allele is not overrepresented in a population of German patients with idiopathic Parkinson's disease
}

\author{
T Gasser, B Müller-Myhsok, A Supala, E Zimmer, G Wieditz, Z K Wszolek, P Vieregge, \\ V Bonifati, W H Oertel
}

Neurologische Klinik, Klinikum Groshadern, Ludwig-MaximiliansUniversität, München, Germany

T Gasser

A Supala

Bernhard-Nocht

Institut für

Tropenmedizin,

Hamburg, Germany

B Müller-Myhsok

Psychiatrische Klinik

der Technischen

Universität München,

Germany

E Zimmer

Paracelsus-Elena

Klinik, Kassel,

Germany

G Wieditz

Section of Neurology, Department of Internal Medicine, University of Nebraska Medical Center, Omaha, NE, USA Z K Wszolek

Neurologische Klinik der Universität Lübeck, Lübeck, Germany

P Vieregge

Dipartimento di Scienze Neurologiche, Università " $\mathbf{L a}$ Sapienza", Roma, Italy

V Bonifat

Neurologische Klinik, Phillips-Universität, Marburg, Germany W H Oertel

Correspondence to: Dr Thomas Gasser Neurologische Klinik, Klinikum Großhadern, Marachioninistrasse 15, 81377 München, Germany. Received 28 September 1995 and in final revised form 22 May 1996

Accepted 3 June 1996

\begin{abstract}
The frequency of the CYP2D6B allele of the gene for debrisoquine 4-hydroxylase was studied in 115 patients with sporadic idiopathic Parkinson's disease, 55 of their healthy siblings, 63 patients with familial Parkinson's disease, 55 unaffected relatives, and 92 patients with Alzheimer's disease and 73 age matched healthy controls. By contrast with several previous studies, no significant variation of allele frequencies could be found between any of the groups studied. The results argue against a significant role of the CYP2D6 gene in the aetiology of sporadic and familial idiopathic parkinsonism in this patient population.
\end{abstract}

\section{(F Neurol Neurosurg Psychiatry 1996;61:518-520)}

Keywords: idiopathic Parkinson's disease; debrisoquine 4-hydroxylase; allelic association

Environmental as well as genetic factors are thought to play a part in the pathogenesis of idiopathic Parkinson's disease. It has been suggested that a variant allele of the gene for debrisoquine 4-hydroxylase (the CYP2D6B allele), which causes a "poor-metaboliser" phenotype, may be more prevalent in patients with both sporadic ${ }^{1-3}$ and familial ${ }^{4}$ Parkinson's disease, but not in patients with multiple system atrophy. ${ }^{5}$ This enzyme is involved in the detoxification of xenobiotic compounds. Although confirmed in several independent studies, the data are not entirely conclusive. Smith et al found a higher proportion of homozygotes for the B-allele in patients with Parkinson's disease. ${ }^{1}$ Armstrong and coworkers found a higher proportion of heterozygotes of the wt/CYP2D6B genotype, ${ }^{2}$ whereas in the study of Kurth and Kurth, both homozygotes and heterozygotes were overrepresented among patients with Parkinson's disease. ${ }^{3}$

We have studied the frequency of the CYP2D6B allele in 115 patients with sporadic Parkinson's disease, 55 of their healthy siblings, 63 patients with familial Parkinson's disease from 34 families, 55 of their unaffected relatives, 92 patients with Alzheimer's disease, and 73 age matched healthy controls.

\section{Methods}

Blood was obtained by venepuncture from consenting subjects and DNA was isolated. Allele status for the CYP2D6B allele was determined by amplifying the respective region of the gene using the polymerase chain reaction. Fragments were digested with BstNI, separated by agarose gel electrophoresis, and detected by ethidium bromide staining, as described previously. ${ }^{1}$

\section{Patients}

Patients with Parkinson's disease were diagnosed according to the United Kingdom Parkinson's disease brain bank criteria. ${ }^{6}$ Their unaffected siblings were questioned for the presence of symptoms of parkinsonism, but not examined neurologically. Familial Parkinson's disease was defined as Parkinson's disease with at least one additional affected person among first degree relatives (from nine of these families, only one affected member was available for DNA studies. In these cases, diagnosis of affected relatives was based on hospital records). Patients with Alzheimer's disease were ascertained from an Alzheimer's disease outpatient clinic by one of us (EZ). Spouses were used as neurologically healthy controls.

All patients with sporadic Parkinson's disease, as well as all spouses, were of white ethnic origin. 68 patients originated from southern Bavaria, the remaining patients from other parts of Germany. Likewise, all patients with familial Parkinson's disease were white; their place of origin however, was more diverse: 11 families (19 patients) originated from southern Germany, 16 families (26 patients) from other parts of Germany. Fourteen patients from five families were ascertained in the United States; their ancestors however were traced to northern Germany (four families) and England. Four patients from two families were from Italy.

\section{Results}

The table shows the results of the genotyping. Total allele frequencies did not differ significantly between any of the groups $(P>0.2$ for all comparisons when applying correction for 


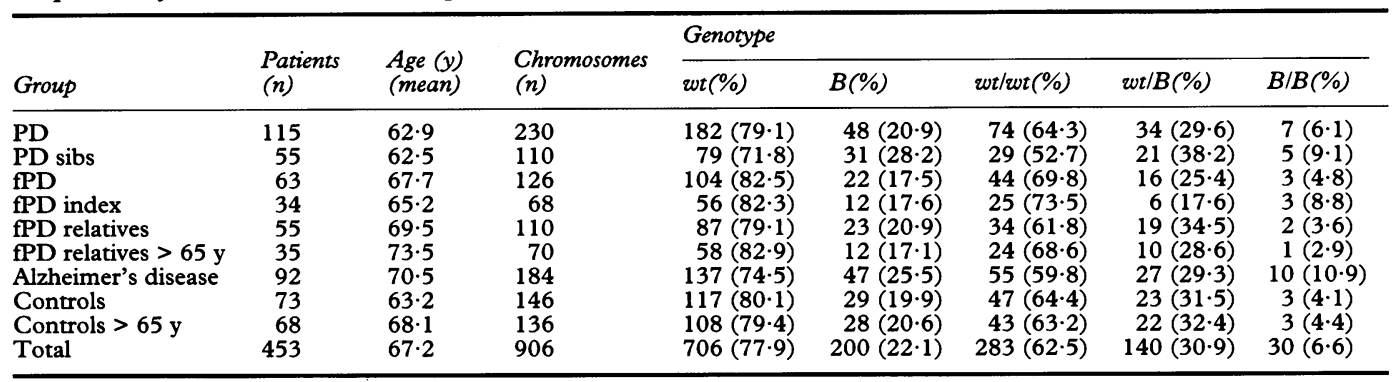

wt = Wild type allele; B = CYP2D6-B allele; PD = Parkinson's disease; PD sibs = healthy siblings of patients with sporadic Parkinson's disease; $\mathrm{fPS}=$ familial Parkinson's disease (at least one additional affected first degree relative); fPD relatives $=$ healthy relatives of patients with $\mathrm{fPD} ; \mathrm{fPD}$-index $=$ index-patients with $\mathrm{fPD}$.

multiple testing, $\chi^{2}$ test). There was no significant difference in the proportion of subjects homozygous for the CYP2D6B allele between any of the groups. If only controls over 65 years of age were included to minimise the possibility of including presymptomatic patients with Parkinson's disease, there was no difference in allele frequencies. The same was true if only index cases of familial cases were analysed. Agundez and coworkers found a higher proportion of wt/CYP2D6B genotypes in patients with Parkinson's disease with onset before 50 years of age. ${ }^{7}$ Among our 40 sporadic patients with onset under 50 years ( 32 to 49 years, mean 42.6 years), the frequency of the B allele (18\%), as well as the proportion of homozygotes, was not different from controls.

\section{Discussion}

By contrast with several previous reports, ${ }^{1-4} 7$ our study did not show a significant difference in the frequency of the CYP2D6B allele between patients with sporadic or familial Parkinson's disease and three different control groups: patients with Alzheimer's disease, healthy siblings, and relatives of patients with Parkinson's disease, and neurologically healthy age matched subjects. The number of patients studied should have been sufficient to detect differences in allele frequencies at a level found in the previous studies (odds ratios between 2 and 3), as a simulation showed a power of more than $95 \%$ to detect a twofold increase in the frequency of the rarer B-allele at a $5 \%$ significance level.

The frequency of the CYP2D6B allele varies considerably among different previous studies. In controls, it ranged from $0 \cdot 10$ to 0.21 , whereas in the Parkinson's disease groups, the proportion of the $\mathrm{B}$-allele ranged from $0 \cdot 22$ to $0 \cdot 36 . .^{23}$ Allele frequencies in our control and Parkinson's disease populations were exactly between affected and unaffected subjects in previous studies.

Several different explanations for these discrepancies are possible:

(1) The results may reflect true differences between German and other populations of patients with Parkinson's disease. This seems unlikely, as there are no known differences in clinical or biographical features between these populations.

(2) As allele frequencies may vary between populations, their ethnic background is of prime importance in association studies. The association reported in previous studies might therefore be due to the selection of the sample. Kurth and Kurth ${ }^{3}$ investigated patients living in the south western United States; their controls were taken from a pool of CEPH families collected in western Europe. Although both populations are white, differences in allele frequencies are possible. Smith and coworkers ${ }^{1}$ did not match their controls for age and sex. It is conceivable that this may cause differences in genetic background, as the ethnic composition of a population, especially in urban areas, may depend on biographical factors.

To avoid these potential pitfalls, we examined unaffected siblings as one of our control groups. Any gene conveying a genetic susceptibility to a disease should be more frequent in affected than in unaffected siblings. ${ }^{8}$ In addition, we selected spouses from patients with Parkinson's disease and Alzheimer's disease as unrelated healthy controls. As most of our patients originated from Bavaria and were referred to our hospital by local physicians, our cohort may be more homogeneous genetically than in previous studies. The group of patients with familial Parkinson's disease is more heterogeneous, as investigators from different countries have contributed cases. Due to low numbers, subgroups among patients with familial Parkinson's disease could not be evaluated.

(3) The increased frequency of poor metabolizer alleles in patients with Parkinson's disease may be a chance finding. The fact that two of the subsequent studies ${ }^{4}$ did not confirm the original association, but found variations in allele frequencies only in subgroups, argues in favour of this explanation. Multiple testing in different subgroups requires an increased level of significance. ${ }^{9}$

(4) Finally, of course, our study may fail to detect an existing association between the CYP2D6B allele and Parkinson's disease, due to insufficient numbers of patients studied, especially if subgroups are analysed.

In a previous study, linkage analysis argued against a mutation in CYP2D6 as a significant determinant in familial Parkinson's disease. ${ }^{10}$ Our results argue against a major role of the CYP2D6B allele in the aetiology of Parkinson's disease in our population, although an effect in a particular subgroup 
cannot be excluded. Allele association studies are hampered by several possible sources of error. ${ }^{11}$ Our study emphasises the need for additional, carefully designed studies when applying this potentially useful method in the search for genetic factors contributing to the aetiology of Parkinson's disease.

The study was supported in part by the Bundesministerium für Bildung und Forschung, Germany (Research program on Barkinson's disease and other basal ganglia disorders, grant No 01KL9001).

1 Smith CAD, Gough AC, Leigh PN, et al. Debrisoquine hydroxylase gene polymorphism and susceptibility to hydroxylase gene polymorphism and susce

2 Armstrong M, Daly AK, Cholerton S, et al. Mutant debrisoquine hydroxylation genes in Parkinson's disease. debrisoquine hydroxylation genes in Parkinson's disease.

3 Kurth MC, Kurth JH. Variant cytochrome P450 CYP2D6 allelic frequencies in Parkinson's disease. $\mathrm{Am} f \mathrm{Med}$ Genet 1993;48:166-8.

4 Planté-Bordeneuve V, Davis MB, Maraganore DM, et al. Debrisoquine hydroxylase gene polymorphism in familial Parkinson's disease. $\mathcal{F}$ Neurol Neurosurg Psychiatry 1994; 57:911-3.

5 Planté-Bordeneuve V, Bandmann O, Wenning G, et al. CYP2D6-debrisoquine hydroxylase gene polymorphism in multiple system atrophy. Mov Disord 1995;10:277-8.

6 Ward CD, Gibb WRG. Research diagnostic criteria for Parkinson's disease. Adv Neurol 1990;53:245-9.

7 Agundez JA, Jimenez-Jimenez FJ, Luengo A, et al. Association between the oxidative polymorphism and early onset of Parkinson's disease. Clin Pharmacol Ther 1995;57 291-8.

8 Schaid DJ, Sommer SS. Genotype relative risks: methods for design and analysis of candidate-gene association studies. Am F Hum Genet 1993;53:1114-26.

Hochberg Y, Tamhane AC. Multiple comparison procedures. New York: Wiley and sons, 1987.

10 Gasser T, Wszolek ZK, Trofatter J, et al. Linkage studies in autosomal dominant parkinsonism: evaluation of seven candidate genes. Ann Neurol 1994;36:387-96.

11 Kidd KK Associations of disease with genetic markers: déjà vu all over again. Am f Hum Genet 1993;48:71-3.

\section{HISTORICAL NOTES}

\section{The Marcus Gunn pupil}

Marcus Gunn (1850-1909) is most often remembered for his description of jaw-winking. The sign consists of widening of a ptosis when the patient chews or opens the jaw. Seen in congenital ptosis, there is an aberrant connection between the innervation of the pterygoids and levator palpebrae. However, Gunn also described an abnormal pupillary response: "dynamic anisocoria" that bears his name.

"It is not sufficient" he says, ${ }^{1}$ "to find that it [the pupil] contracts well or fairly well on exposure; the eye must also be kept under direct stimulation of light and the pupil watched as to whether or not it shows that secondary dilatation under continued exposure that is found associated with the amblyopia of retro-ocular neuritis. If the vision of one eye only is affected, it is important to compare the behaviour of the two pupils when stimulated directly or consensually. Thus, in partial affection of the right optic nerve the right pupil will show this secondary dilatation during continued exposure to direct stimulation, while the left pupil will show the same behaviour on consensual stimulation. On the other hand, on stimulation of the left eye both the right and left pupil will behave normally. I need not remind you of the importance of this observation, inasmuch as it not infrequently enables us to diagnose a retro-ocular neuritis in the absence of all ophthalmoscopic evidence."

Levatin's "swinging flashlight test for pupillary escape" 2 is a modification of Gunn's technique. However, the appropriate speed of swinging the light from one eye to the other has to be found by trial and error; and, the procedure is unreliable in the presence of bilateral afferent defects of light conduction. ${ }^{3}$

The Marcus Gunn pupil in effect is an adaptation of the light reflex during persistent stimulation. It corresponds to the decrement of evoked axonal potentials in the optic nerve of the rabbit that follows after a biphasic response to light (figure) ${ }^{4}$ In afferent lesions the input of the residual stimulus that normally triggers pupillary constriction after several seconds of continued stimulation is reduced and approximates to the backgro: nd light. The pupil again dilates.

Born in Culgower in Sutherland, Marcus Gunn, a contemporary of Robert Louis Stevenson at Edinburgh, graduated aged 23. His fascination with ophthalmology was aroused by Walker and Argyll

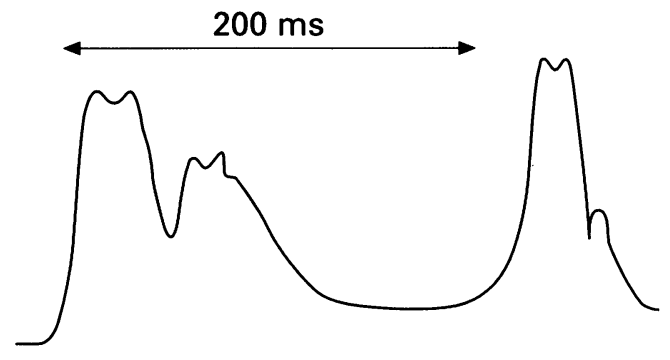

Optic nerve response to full field flash for $200 \mathrm{~ms}$ (arrow). Biphasic "on" response followed by low voltage sustained activity, and final brief "off" wave. Modified from Bartley and Bishop. ${ }^{4}$

Robertson. After a brief visit to Moorfields Eye Hospital, he studied comparative anatomy at University College, London. Returning to Scotland, at a Perth asylum, single handed, he produced a series of relatively original observations of the fundus using the recently developed direct ophthalmoscope. (It was not until 1897 that Gowers' Medical Ophthalmology was published as the first major atlas of the fundus as revealed by direct ophthalmoscopy). A visit to Vienna in 1874 brought further training with Jaeger. Moorfields again drew him to London, where he improved medical records. He managed to reduce the sepsis rate of cataract surgery by implementing Lister's aseptic techniques. After a visit to Australia he became the senior surgeon at Moorfields. He made outstanding observations of the human retina and vessels, describing the poor prognosis of soft exudates in "albuminuric retinitis". A keen student of nature, Gunn collected the fossils of plants and fish of the Jurassic period from sandstone rocks. Many of his specimens were retained by the British Museum.

$$
\begin{array}{r}
\text { JMS PEARCE } \\
304 \text { Beverley Road, } \\
\text { Anlaby, Hull, } \\
\text { East Yorks, HU10 } 7 B G, U K
\end{array}
$$

1 Gunn RM. Functional or hysterical amblyopia. Ophthalmol Reviews 1902;21:271-80.

2 Levatin P. Pupillary escape in disease of the retina or optic nerve. Arch Ophthalmol 1959;62:768-79.

3 Landau WM. The Marcus Gunn phenomenon: loose canon of neuro-ophthalmology. Neurology 1988;38. 1141-2.

4 Bartley SH, Bishop GH. Some features of the optic nerve discharge in the rabbit and the cat. $\mathcal{f}$ Cell Comp Physiol 1942;19:79-93. 